- Clarification of some of the terminology and concepts relating to occlusion as it is used in everyday practice, making clear why these concepts matter

- Undertaking a simple pre-operative examination of the occlusion as a matter of routine

- Helping clinicians identify cases where articulated study casts will help plan treatment and design restorations

- Advice is provided about selecting an appropriate articulator and taking appropriate records at the treatment stage.

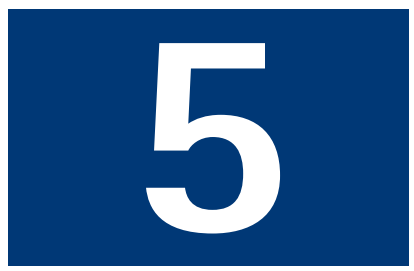

\title{
Crowns and other extra-coronal restorations: Occlusal considerations and articulator selection
}

\author{
J. G. Steele ${ }^{1}$ F. S. A. Nohl ${ }^{2}$ and R. W. Wassell ${ }^{3}$
}

\begin{abstract}
For many dentists, occlusion carries an air of mystique. It even seems sometimes that a perverse pleasure is derived in making the whole subject more complicated than it really is. As a clinician, you need to be able to decide what you expect from your proposed restoration, and to identify situations where you may need to alter the existing occlusal scheme. At a fundamental level, you also need to provide the laboratory with appropriate clinical records to ensure that when you fit them, adjustments to the expensively prepared restorations are minimal. This requires a sound understanding of the basics.
\end{abstract}

CROWNS AND EXTRA-CORONAL RESTORATIONS:

1. Changing patterns and the need for quality

2. Materials considerations

3. Pre-operative assessment

4. Endodontic considerations

5. Jaw registration and articulator selection

6. Aesthetic control

7. Cores for teeth with vital pulps

8. Preparations for full veneer crowns

9. Provisional restorations

10. Impression materials and technique

11. Try-in and cementation of crowns

12. Porcelain veneers

13. Resin bonded metal restorations

1"3Senior Lecturer in Restorative Dentistry, Department of Restorative Dentistry, The Dental School, Framlington Place, Newcastle upon Tyne NE2 4BW

${ }^{2}$ Consultant in Restorative Dentistry, The

Dental Hospital, Framlington Place,

Newcastle upon Tyne NE2 4AZ

${ }^{*}$ Correspondence to: J. G. Steele

E-mail:jimmy.steele@ncl.ac.uk

\section{Refereed Paper}

@ British Dental Journal 2002; 192: 377-387
This fifth article in the series will try to present important occlusal concepts in a way which relates directly to the provision of successful crowns. It is not a comprehensive guide to occlusion, or a manual of techniques for extensive fixed prosthodontics. There are several useful books and articles dedicated to the subject and some of these are specifically referenced (if a technique is particularly well described) or are listed in the further reading section. However, we hope that this article should allow you to avoid most of the problems associated with the provision of crowns. Occasionally some pre-operative occlusal adjustment is needed. Our experience is that this is best taught 'hands on' and we would recommend attending an appropriate course before attempting more complex adjustments.

\section{BASIC CONSIDERATIONS - WHAT MATTERS?}

One of the essential starting points with occlusion is to make sure that the terminology is clear. There are any number of occlusal terms, many of which overlap. There are only a few that really matter and these need to be understood if what is to follow is to make any sense.

\section{The intercuspal position (ICP or IP)}

Synonyms: centric occlusion (CO), maximum intercuspation

\section{What is it?}

Most dentate patients, when asked simply to 'bite together on your back teeth', close immediately into a comfortable, reproducible "closed" position where the maximum number of tooth contacts occur. This is the intercuspal position
(ICP). Travel into this position is partly guided by the shape of teeth and partly by conditioned neuromuscular co-ordination. ${ }^{1}$ ICP is the most 'closed' position of the jaws.

\section{Why does this matter?}

ICP is usually the position in which vertical occlusal forces are most effectively borne by the periodontium with teeth likely to be loaded axially, which helps to stabilise their position. Indeed it is the end point of the chewing cycle where maximum force is exerted. In everyday practice this is the position of the jaws in which restorations are made.

\section{Guidance (from the teeth) \\ What is it?}

When a patient moves their mandible from side to side so that the teeth in opposing jaws slide over each other, the path taken is determined partly by the shapes of the teeth which make contact, as well as by the anatomical constraints of the temporomandibular joints (TMJs) and masticatory neuromuscular function. Each has a bearing on the other, and, for want of a better term, they should work in harmony. In these circumstances the teeth provide guidance for the movement of the mandible. The shape and form of the temporomandibular joints also guide the movement of the mandible (sometimes called posterior guidance). Guidance teeth can be any teeth, anterior or posterior.

When the patient slides the mandible out to one side, the side they move the mandible towards is called the working side (because it is usually the side on which they are about to 
Fig. 1 Right canine guidance during right lateral excursion. Posterior teeth are discluded but contact remains between the lateral incisors

Fig. 2 Left canine guidance during left lateral excursion. Here the canines are the only teeth in contact

Fig. 3 Shared contact between many posterior teeth in right lateral excursion. Guidance is described as being group function

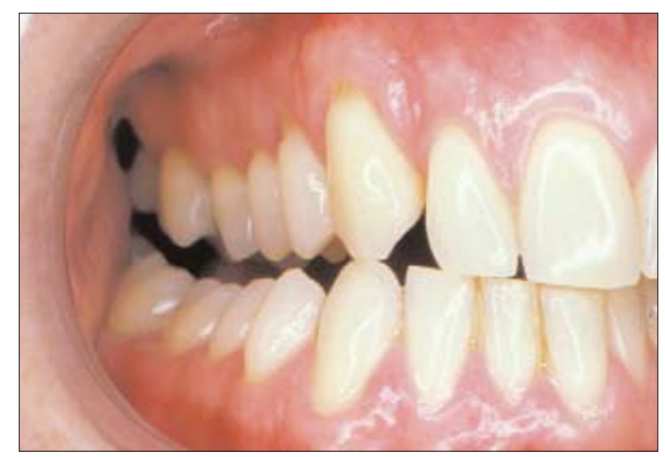

ture or decementation, particularly if these loads are heavy. Other manifestations of problems with guidance include:

- Fractured teeth or restorations

- Accelerated local wear

- Tooth migration

- Tooth mobility

- TMJ dysfunction

To avoid these it is important to identify which teeth provide guidance before you start tooth preparation. If the guidance is satisfactory, and the guidance tooth or teeth are strong enough to withstand the likely loading in the long term, it is usually best to try to re-establish the same guidance pattern in the new restoration. Techniques for doing this are described later in the article. Occasionally, you may feel that a tooth that you are about to crown is insufficiently robust to carry a guidance contact and the guidance is best moved onto other teeth. A specific example of this would be where a broken down guidance canine is restored with a post retained crown. There may be a risk of root fracture of the tooth in the longer term because of the heavy lateral forces. In a case like this, by taking a little care with the shape of both preparation and crown, guidance can often be transferred from the canine to the premolars, if they are in a better position to accept the heavy loads.

Other practical reasons for identifying guidance teeth include:

- The need to provide clearance from the opposing tooth during preparation, not just in ICP, but also along the guidance track. If you do not do this you can end up unwittingly transferring all the guidance forces on to your new crown.

chew). The other side, the side the mandible is moving away from, is called the non-working side. So for example, an excursion to the right (as may occur during chewing) will make the right side the working side and the left the nonworking side, whilst during an excursion to the left the reverse will be true. During these excursions the upper and lower guidance teeth will be in contact and partly dictate the movement of the mandible. Canine guidance is where the upper and lower canines on the working side are the only teeth in contact during a lateral excursion, causing all of the posterior teeth to disclude (Figs 1 and 2). When several pairs of teeth, usually premolars or premolars and canines (and sometimes molars) on the working side share the contacts during excursions group function is said to take place (Fig. 3). Other patterns of guidance can take place, using almost any combination of teeth.

Incisors and canines usually provide protrusive guidance, when the mandible slides forward, but where there is only a limited overbite the posterior teeth may be involved.

Why does it matter?

Guidance teeth are repeatedly loaded non-axially (laterally) during excursions. As a result heavily restored or crowned teeth may be at risk of frac-
- The need to select and prescribe an appropriate material to restore the guidance surface (metal is usually best if possible).

These aspects are discussed in detail later in the series.

The message is that getting guidance right is one of the most important aspects of crown provision; problems can, and will, occur unless guidance is correctly managed on teeth to be crowned.

\section{Interferences}

What are they?

Interferences are any tooth-to-tooth contact(s), which hamper or hinder smooth guidance in excursions or closure into ICP. An interference on the side to which the mandible is moving is called a working side interference. An interference on the side from which the mandible is moving is called a non-working side interference (NWSI) or balancing side interference. There is a distinction to make between NWSIs and non-working side contacts: in the latter case, excursions are guided equally by working and non-working tooth contacts, akin to the balanced articulation often taught as an ideal complete denture occlusion. However, where 
there is a NWSI it acts as a cross arch pivot, disrupting the smooth movement and separating guidance teeth on the working side (Fig. 4).

\section{Why does it matter?}

There has been much written about the significance or otherwise of interferences, particularly NWSIs, in relation to initiating parafunction and TMJ dysfunction. Warnings of the direst consequences to the stomatognathic system and beyond from NWSIs are frankly misleading though. Many people function perfectly happily with a mouthful of NWSIs. However, when contemplating crowns there are important implications. Most NWSIs are on molars so teeth or restorations directly involved are subject to high and often oblique occlusal forces with the consequent risk of fracture or uncementing.

As a general rule, it is best to remove interferences before tooth preparation if the interference is on a tooth which is to be prepared. This applies to all types of interference - working, non-working and protrusive. In practice it is best to do this at a separate appointment prior to tooth preparation. This will allow the patient time to adapt to a new pattern of excursive guidance, and you time to refine the guidance if necessary. The process of dealing with a non-working side interference prior to preparation is shown in Figure 4. If you do this, it is important to identify suitable teeth on the working side to take over the guidance once the interference has been eliminated. If there are no teeth to take over the guidance, it may be impossible to eliminate the NWSI. If you are in any doubt it would be best to seek advice before cutting the tooth.

Where interferences exist on teeth that are not themselves to be prepared, the need for adjustment may be less important. Many people have asymptomatic interferences and seem to be able to lead a normal existence and we certainly would not advocate the removal of all interferences as a public health measure.

One final point is that it is disturbingly easy to introduce new interferences when you place restorations, even where there were no interferences previously. If you check your preparations for adequate clearance, not only in ICP but in lateral and protrusive excursions as well, the chances of this occurring should be minimised. Obviously, there is the opportunity to remove minor interferences on the final restoration before cementation.

\section{Retruded Contact Position (RCP)}

Synonyms: centric relation (CR), centric relation contact position (CRCP), retruded axis position (RAP), terminal hinge position.

\section{What is it?}

This is the position of the mandible when the first contact between opposing teeth takes place, during closure on its hinge axis (or retruded arc of closure), that is with the condyles maximally seated in their fossae. This condylar position is one of health. Generally, as the mandible hinges
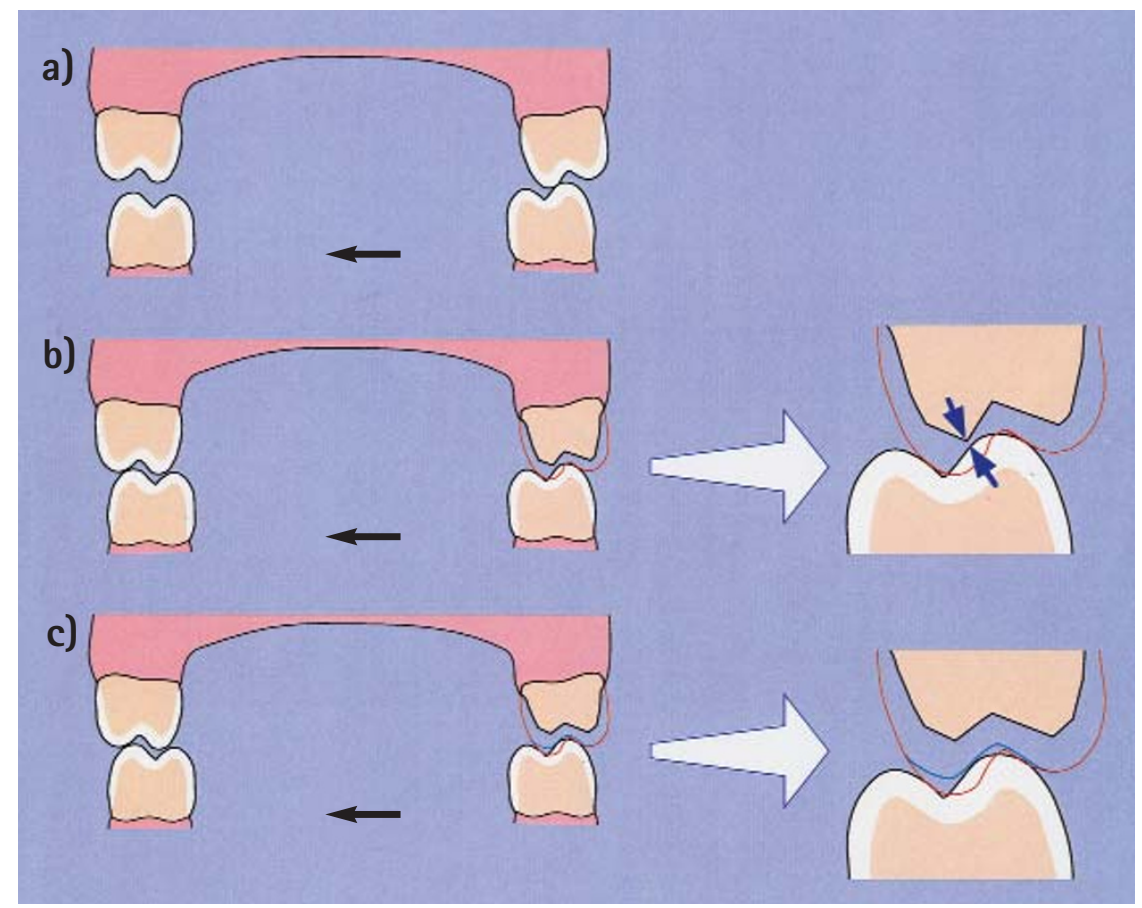

Fig. 4 A non-working side interference between the left first molars and the possible consequences of carrying out crown preparation without appreciating its presence (transverse section): a) During a right lateral excursion (see black arrow) the left first molars act as a cross-arch pivot lifting the teeth out of contact on the working side; b) The maxillary first molar has been prepared for a crown. Occlusal reduction has eliminated the pivot, allowing the teeth on the working side to contact during lateral excursion. However, clearance between the preparation and opposing teeth is now inadequate which may cause problems with the provisional restoration. Worse still, the definitive restoration may require gross adjustment resulting in its perforation; c) You can avoid these problems by removing the non-working side contact prior to tooth preparation (blue line represents tooth recontoured in this way)

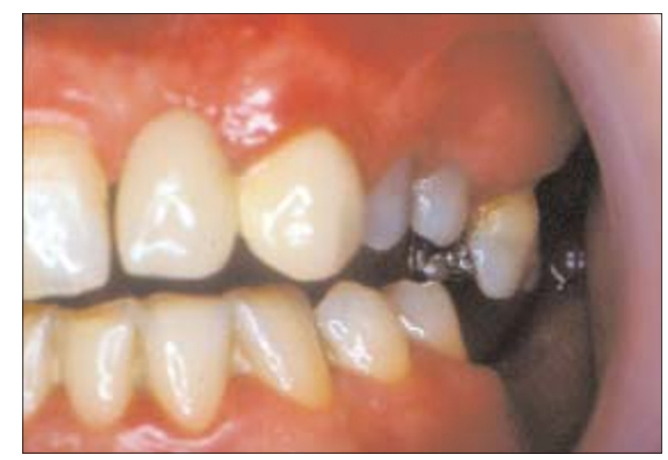

closed with the condyles in this position, there is a contact between a pair of teeth somewhere around the mouth (Fig. 5). The mandible will then close, from this retruded contact, down into ICP, usually sliding forward and laterally (Fig. 6). If you want to try to manipulate a patient's mandible into this position it is important that they are relaxed (Fig. 7), otherwise it can be very difficult and you will feel resistance to free movement of the mandible. For about $10 \%$ of people ICP will be the same as $\mathrm{RCP}^{2}$ and in these cases if you hinge the mandible until the teeth are in contact they will go straight into ICP with no deflective contact.

\section{Why does it matter?}

There is no magic quality about RCP, but there are a number of reasons why RCP and the associated slide into ICP may be relevant when providing crowns. Box 1 contains a more detailed
Fig. $5 \mathrm{~A}$ retruded contact between maxillary molar and mandibular premolar. Most retruded contacts cause no problems. This one resulted from over-eruption of the molar and the interference was associated with accelerated wear of the incisors 


\section{BOX 1: RCP AND CROWNS}

Most crowns and other extra-coronal restorations will be made to conform with the patient's ICP and usually a slide from RCP to ICP will be of no major relevance when providing crowns. In some circumstances additional management may be appropriate. The following are the situations where adjusting the contact in RCP is likely to be most important:

1. As a general rule, when RCP involves a tooth you are about to prepare it is often best to remove the deflective contact at an appointment before you start tooth preparation.

2. When re-organising the occlusion at a new vertical dimension you really have no option but to construct the new occlusion, if not at RCP itself, certainly around centric relation (with the condyles in the hinge axis). This represents the only reproducible starting point.

3. If you need space but you wish to avoid increasing the vertical dimension, there may be the scope to 'distalise' the mandible to create space lingually for anterior crowns (only possible where there is a bodily translation between RCP and ICP).

4. If you are about to restore anterior teeth and the RCP contact results in a strong anterior thrust against the teeth to be prepared. Although we would usually advise removal of a deflective RCP interference before preparation if it is on a tooth you are about to prepare for a crown, many dentists do no not carry out any such adjustment and no problems result. This is probably because cutting the crown preparation effectively removes the contact. By removing it before preparation though you can ensure sufficient removal of tissue to allow space for the crown without re-introducing it in an uncontrolled way when the restoration is made. The principles involved are the same as those for removing non-working side interferences (see Fig. 4).

Fig. 6 The slide from retruded contact position (RCP) to the intercuspal position (ICP). Here, in RCP the molars make first contact as shown by the arrows. The magnitude and direction of the slide can be estimated at both the molar and incisor regions
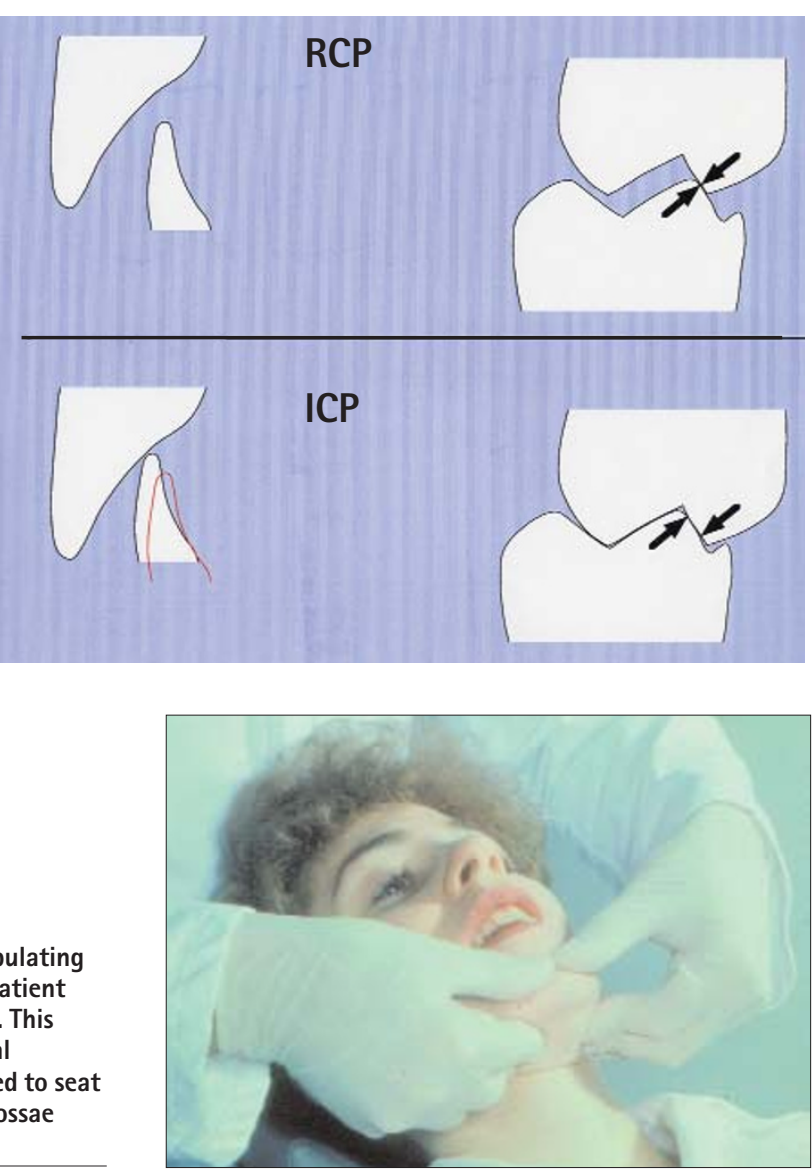

analysis of the situations where it may be a problem. In many, but not all cases, managing RCP is of less significance than managing guidance effectively, but there are times when adjustments need to be made and, clearly, it is always better to identify potential problems than blindly to hope for the best. To this end it is always worth examining RCP pre-operatively so that you at least know where it is. On the very few occasions where it is likely to be a major factor affecting your restorative procedure, casts mounted in RCP (or in the hinge axis just before the teeth actually contact) on a semi-adjustable articulator will facilitate occlusal examination and allow trial adjustments.

Establishing and recording RCP, and particularly re-organising the occlusion, are often difficult and we would refer you to Further Reading below if you wish to follow these subjects up in more detail.

\section{PRACTICAL ASPECTS OF OCCLUSION: RECORDS FOR PLANNING CROWNS}

\section{Records for planning crowns: The occlusal examination}

Before providing crowns it really is mandatory to undertake some sort of an occlusal examination. The following observations take moments to gather and are worth the effort:

- Check ICP (for reproducibility and contacts on the teeth to be restored)

- Check RCP (to establish whether there is any deflective contact or slide which you ought to know about). For technique see Reference 3.

- Check the relationship of the teeth in lateral and protrusive excursions (to determine whether your crowns will be involved in guidance or if you need to consider removing an interference).

A more detailed and lengthy examination, where all of the contacts are marked using good quality ultra thin articulating tape (Fig. 8) is sometimes indicated, particularly where there has been a history of temporomandibular dysfunction or where there is a specific occlusal problem which you need to address. The various components, which may be included in a full occlusal examination, are given in Box 2.

\section{Records for planning crowns: hand-held study} casts

Hand-held casts can be a very useful aid to examination and treatment planning. To glean maximum information from them they 
need to be made from good quality impressions, which have been handled and poured correctly. We will return to this important but underrated subject later (see below: 'Opposing Casts').

Hand-held study casts enable:

- A judgement to be made regarding the ease of obtaining a stable ICP. This helps to determine whether or not an interocclusal record is required for the working casts upon which the restoration(s) will be made.

- An unimpeded view of ICP. It is possible to view aspects such as the lingual, which it would not be possible to see at the chairside.

- Careful evaluation of clinical crown height and the availability of inter-occlusal space for restorative material. These two factors can help make the decision on how to facilitate the restoration of short teeth (see Part 3 'Pre-operative assessment' in this series).

However tempting it may be to assume otherwise, hand-held casts provide no information about excursive tooth contacts or RCP, beyond the distribution of wear facets.

\section{Records for planning crowns: Articulated study} casts

Accurate casts of the dental arches mounted in a semi adjustable articulator are the most important tools of the trade when constructing artificial crowns. The need for an articulator and the positions in which you mount the casts depend on what you need to do (see Box 3). Articulators are surrounded by an aura of mystery, but at the end of the day they are a tool to help give your patient a successful restoration and to help you to save time, money and hassle. The quality of the final result is much less dependent on the articulator you use than it is on the care you exercise to make and mount the casts that you put in it.

There is little merit in examining study casts for planning purposes on a simple hinge or other 'non-anatomical' articulator because the ability to replicate physiological movements will be, at best, crude, and at worst, wholly misleading. A non-anatomical articulator will allow casts to be put into a reproducible ICP, which may be helpful if there are insufficient contacts to make handheld casts stable, but that is the limit of what a simple hinge articulator can do.

\section{BOX 2: COMPONENTS OF AN OCCLUSAL EXAMINATION}

A full occlusal examination including all of the components below is rarely indicated, but different components may be required at different times. Visual assessments of ICP, RCP and guidance teeth are always indicated where you are planning crowns. The following may also be useful:

1. Examining for signs of temporomandibular dysfunction:

- Palpation of muscles of mastication for tenderness.

- Palpation of the temporomandibular joints and detailed assessment of any clicks or deviations in mandibular movement.

- Assessment of the range of mandibular movement.

2. Checking for facets, fremitus, mobility and drifting (particularly where there are problems with individual teeth).

3. Marking up of the dry teeth in different coloured tapes (Fig. 8) to show contacts in all excursions using a high quality articulating tape (where there are issues affecting the entire occlusal scheme).

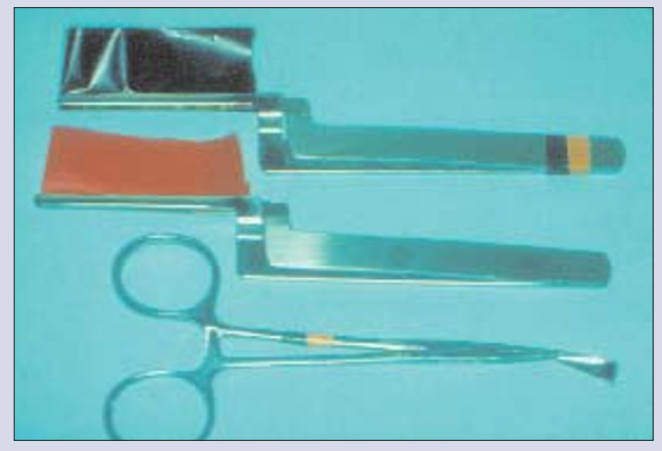

Fig. 8 Tools for examining occlusal contacts: foil shimstock held in forceps and high quality ultra-thin articulating tapes held in Millers forceps

\section{BOX 3: WHEN TO USE A SEMI-ADJUSTABLE ARTICULATOR DURING THE MANUFACTURE} OF CROWNS

1. If you wish to ensure appropriate guidance with your new restorations, particularly where multiple crowns are involved.

2. If you plan to increase the vertical dimension at all.

3. In any case where either you are going to remove so many of the occlusal contacts that ICP will effectively be lost and/or where you plan to make a new ICP based on RCP (sometimes known as a reorganised occlusion).

4. Where you plan to remove occlusal interferences (the study casts can enable a trial adjustment to be carried out).

5. When there is a need to provide an occlusal stabilisation appliance (occlusal splint), either before treatment to stabilise jaw position or after treatment to protect restorations from the effects of bruxism. 


\section{BOX 4: TAKING A GOOD OPPOSING IMPRESSION}

1. Choose stock impression trays with adequate rigidity which, perhaps in combination with an adhesive, have sufficient retentive features to hold set alginate securely.

2. If the restoration is on a very posterior tooth you may need to extend the tray with a rigid material (green stick or compo but not carding wax) to ensure that the alginate is supported in important posterior areas.

3. Make sure adhesive has been air dried in the tray before loading alginate. Lots of wet adhesive acts as a lubricant not an adhesive, and if the alginate pulls away from the tray you are left with an enormous but not very obvious distortion in the final cast.

4. Dry the teeth with gauze or cotton wool to remove saliva, which aids tremendously in recording occlusal detail and producing of a smooth, hard cast surface. It can be done as the alginate is being mixed.

5. Smear a little alginate firmly onto the dry occlusal surfaces with your finger as the assistant finishes loading the tray.

\section{After removal}

6. Check to make sure the material has not pulled out of the tray (Fig. 9). If it has, retake it and do not assume it will be all right or be tempted to press dislodged material back into place; you will pay in the time spent adjusting the final crown.

7. Cut off the heels of the impression flush with the tray if you do not need them, they are prone to distort when you lay the impression down on the bench or even when it is loaded with wet stone. This takes seconds.

8. If you get the chance, check the casts, if not, ask the technician to do this and to flick off any air bubbles on critical surfaces (Fig. 10).

9. In really critical cases, consider using silicone to obtain a very accurate opposing cast especially if there is to be a delay before pouring up.

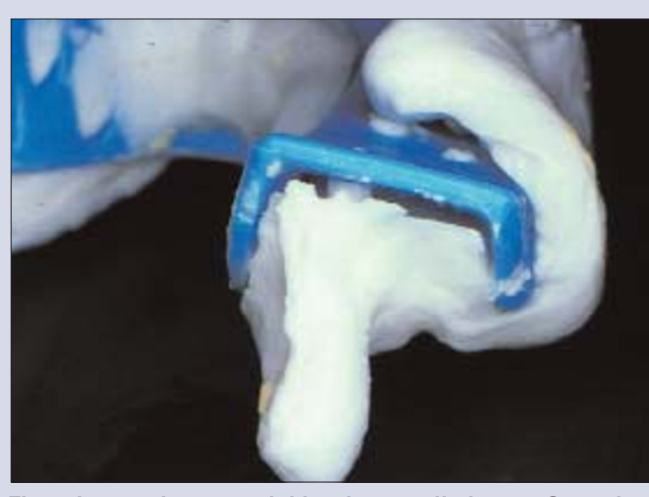

Fig. 9 Impression material has been pulled away from the tray on withdrawal from the mouth.

This will equate to at least a $1 \mathrm{~mm}$ occlusal error

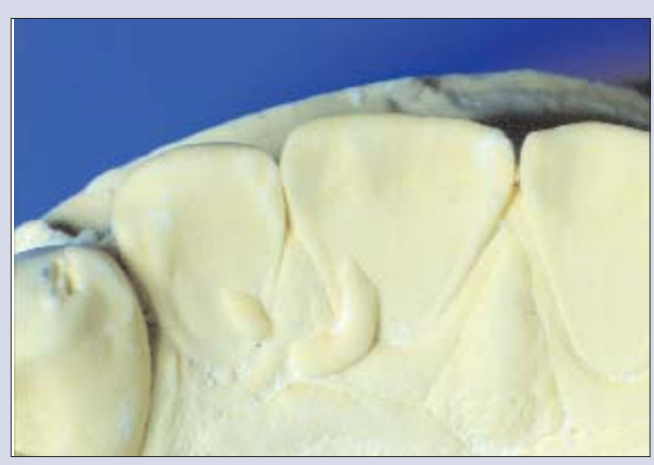

Fig. 10 Large stone blebs on the palatal surfaces of upper anterior teeth as a result of air bubbles in the impression
The combination of a facebow record (which locates the approximate position of the condylar hinge axis in relation to the upper arch) and a jaw relation record (which then locates the lower cast to the upper), enables movements of casts articulated on a semi-adjustable articulator to be reasonably anatomical. You can simulate the movements of the teeth in lateral and protrusive excursions, and around the hinge axis and be confident that what you see is close to what is really happening in the mouth. However, whilst the instrument is key, the quality of the casts and the care with which they are mounted are just as critical. There is no room for carelessness at this stage, wrongly articulated casts are probably worse than no casts at all as they may result in false assumptions about treatment. Similarly, inaccuracies with the original impressions can result in profound errors and the use of an accurate and stable impression material (such as addition cured silicone) may be appropriate in cases where a detailed occlusal analysis is necessary. Details of how to record a facebow record and a retruded hinge axis inter-occlusal record can be found in References 3 and 4. Some simple tips on accurate impression recording can be found in Box 4 .

Although you can see and reproduce movements with carefully articulated casts, you may often want to go on to the next stage and prepare a diagnostic wax up.
Records for planning crowns: the diagnostic wax-up

In addition to its uses in planning changes in appearance (see Aesthetic Control - the sixth article in this series), a diagnostic wax-up can be an absolutely invaluable technique where you are changing the occluding surfaces of several teeth with crowns or resin bonded restorations and allows you to plan the following:

- The new static occlusal contacts (in ICP) and the shape of the guidance teeth

- The impact that the modified occlusion has on appearance

- The best options for creating interocclusal space for restoration(s) or optimising crown height by periodontal surgery (see Part 3: 'Pre-operative Assessment' in this series).

You can also use the completed wax-up as a template to determine the form of temporary and final restorations.

\section{PRACTICAL ASPECTS OF OCCLUSION: RECORDS FOR MAKING CROWNS}

When the diagnosis stage has been completed, the crowns or restorations still need to be made, and various records are essential at this stage too. This section discusses the choice of articulator and the need to obtain accurate occlusal records, including the simplest things such as 
opposing impressions, which are a frequent source of error. Finally, it introduces ways of controlling guidance on front teeth.

\section{The articulator}

When manufacturing the final crowns, in the interests of simplicity and cost it would seem sensible to use the simplest cast relating device that will not compromise the final restoration. ${ }^{5}$ Small numbers of restorations, which are not involved in excursive contacts, can very reasonably be made on a non-adjustable articulator and then any adjustments made in the mouth before final cementation. However, crowns involved in excursions benefit from the use of an articulator with anatomical dimensions so that the excursive movements can be made and the shape of the crown adjusted in the lab with reasonable accuracy, saving chairside time. This becomes particularly important, and cost effective, when several restorations are being created at the same time. Highly sophisticated semiadjustable and fully adjustable articulators are available for this purpose, but the majority of cases can be managed quite satisfactorily using a less sophisticated, fixed average value articulator in combination with a facebow.

It may not be possible to check occlusion on adhesive restorations prior to cementation, either because the act of checking may damage porcelain, which is delicate until cemented, or because they will not stay in place during excursions. In these cases, controlling the role of the restorations in guidance can be critical to their long-term survival. A semi-adjustable articulator can be invaluable in situations such as these because it allows the technician to secure restorations onto the working cast and do the critical adjustments in the lab so that all you need to do is cement them with little or no adjustment afterwards.

\section{Opposing casts}

In any discussion about articulators, it is disturbingly easy to forget the importance of an accurate cast to oppose the working cast. The opposing impression is often the last thing we do and, after a long session preparing teeth, making temporaries and taking impressions it tends to be a bit of an afterthought. However, a poor opposing impression is very easy indeed to achieve and yet can cost a great deal of precious time subsequently. A cast made with a distorted impression or a porous impression resulting in plaster blebs on occlusal surfaces will not fit comfortably into ICP. If such a cast is used in the lab it can result in a crown which looks perfectly good on the cast but which may be very high in the ICP and which can take a great deal of time to get right prior to fit. It is easy to record bad opposing impressions, but good ones are just as easy. Attention to the few steps listed in Box 4 takes, literally, no extra time but can save a lot of heartache. In an ideal world every opposing impression would be recorded in a dimensionally accurate and stable material such as an addition cured silicone, but this is probably not economically realistic. Box 4 describes the use of alginate for an opposing impression. In cases involving multiple restorations though, a very stable and accurate material may be cost effective in the long term.

\section{Interocclusal records (IOR)}

Once you have your working impression and opposing impression you then need to decide whether you need to provide additional information to the lab to allow them to mount the casts; an inter-occlusal record (IOR). There is a common perception that providing an intercuspal record (such as a wax or silicone 'bite') will improve the accuracy of mounted casts. The truth is that in many cases it does precisely the opposite. ${ }^{6}$

For a patient with a stable intercuspal position, the loss of interocclusal contact created by preparation of a tooth for a single unit restoration, is unlikely to detract from the ease with which working and opposing casts can be located in ICP. In this circumstance, placing a layer of wax or silicone between the casts to help to locate them can often result in them failing to seat into ICP at all, and there is a very serious risk of the record introducing inaccuracies, rather than acting as the 'insurance policy' you intended. It is worth taking the opportunity of examining the ease with which any study casts can be located by hand before deciding whether an IOR is needed. Often (perhaps even usually) you are better with nothing at all.

Sometimes an IOR is required to stabilise casts, particularly where the teeth that are prepared are key support teeth in an arch. The choice of materials is generally between hard wax alone, hard wax (as a carrier) used with zinc oxide/eugenol, silicone elastomers and acrylic resins. The fundamental requirement is to obtain enough detail in a dimensionally stable recording material to enable casts to be confidently located in the laboratory whilst not recording so much detail that it stops the casts seating. Occlusal fissure patterns reproduced accurately in the IOR may well not be reproduced to the same extent in the cast, preventing full seating of the casts in the record. Furthermore, an IOR which contacts soft tissues in the mouth and causes their displacement (which is obviously not reproduced in the stone cast) will result in an IOR which will not seat accurately (Figs 11 and 12). In order to meet the requirements for success, an IOR should:

1. Record the tips of cusps or preparations BUT

2. Avoid capturing fissure patterns as much as possible

AND

3. Avoid any soft tissue contact

The key to a successful record is not so much the type of material used, but how it is used. The smaller the amount you use, the less it is likely to cause a problem. A small, trimmed record, restricted to the area of the preps themselves, 
Fig. 11 A very accurate full arch occlusal record in a silicone material. The excessive detail may well hinder seating on a stone cast. See Fig. 12

Fig. 12 The combination of a very detailed silicone occlusal record and less detailed stone cast, particularly of the occlusal fissures, has meant that the record will not seat. There were sufficient tooth contacts in the intercuspal position not to need an interocclusal record for this case!
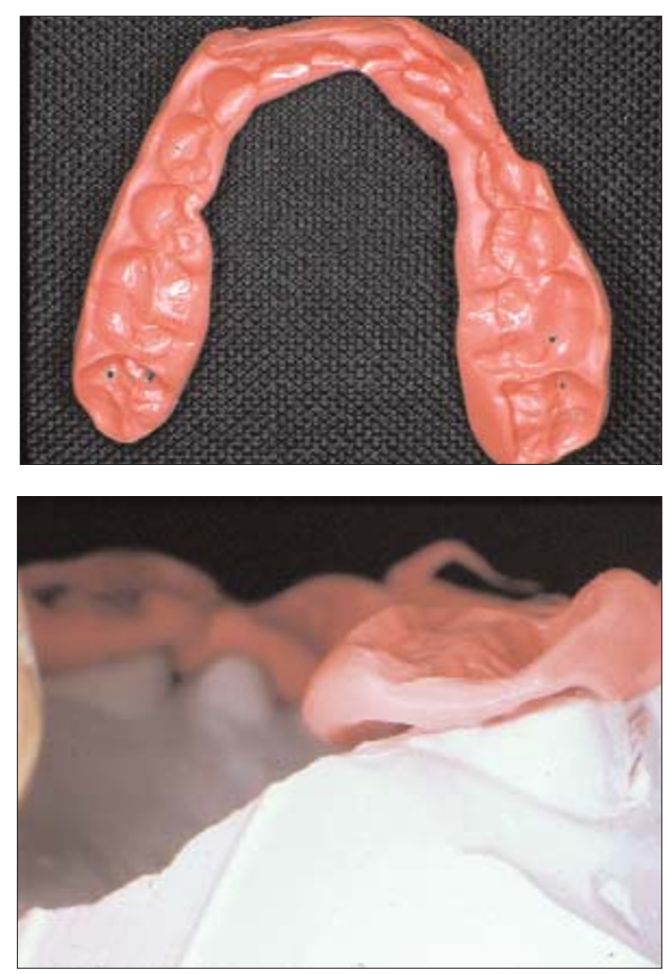

with all interproximal tags and undercuts removed is the ideal. It is usually necessary to trim the record to achieve these requirements and silicone materials trim very easily with a scalpel (Fig. 13). One final consideration is that stone casts and dies can easily be abraded by IORs especially those made of acrylic resin and special care is required in the laboratory when these materials are used in combination.

Whether or not you have used an interocclusal record, you can easily verify the accuracy of mounted casts in the ICP using foil shimstock. When held tightly together the cast should hold the shim between the same teeth as they do in the mouth.

\section{Copying tooth guidance}

When a restoration is being provided which includes a guiding surface for mandibular excursions (lateral, protrusive or anything in between), the technician needs to know what form the contacting surface is to take. Failure to appreciate this risks introducing excursive contacts that are not in harmony with the other guidance teeth, the condylar movements and musculature. This is discussed fully earlier in the article, but common manifestations of disharmony include:

- Decementing and fractured crowns

- Tooth mobility

- Tooth migration

- TMJ dysfunction

Because they are intimately involved in speech formation, getting guidance wrong on the palatal surfaces of upper anterior teeth may also come to light as phonetic problems.

The guidance of a new restoration can often be made simply to fit in with that provided by adjacent teeth (for example in group function). In this case the new functional surface is relatively straightforward to achieve on a semi-adjustable articulator or even at the chairside. However, if several teeth are to be prepared there may be no existing guiding surfaces left intact after preparation, so all clues to guidance are lost (Fig. 14). Where satisfactory guidance is present before you start, there are several ways of copying it before you prepare the teeth. A commonly used technique is to use a putty matrix made on a cast of the tooth surfaces to be copied, but this technique will often not provide the tight control over tooth shape which is required. The two most effective methods to address this problem necessitate the use of a facebow and semi-adjustable articulator to allow anatomical movements in excursions. They are:

1. The 'crown about' method: Alternate teeth are restored, thus maintaining the shapes of functional surfaces, which continue to provide guidance for the articulated casts. This technique is well described in Reference 7.

2. The custom incisal guide table: This is another way of copying satisfactory anterior guidance from teeth or trial restorations that provide crucial excursive contacts. Unlike the previous method, it enables guidance to be copied for just one restoration. It involves moving study casts (on a semi-adjustable articulator) through the full range of lateral and protrusive excursions with a mound of un-set acrylic on the guide table, so that the tip of the articulator guide pin shapes the acrylic dough

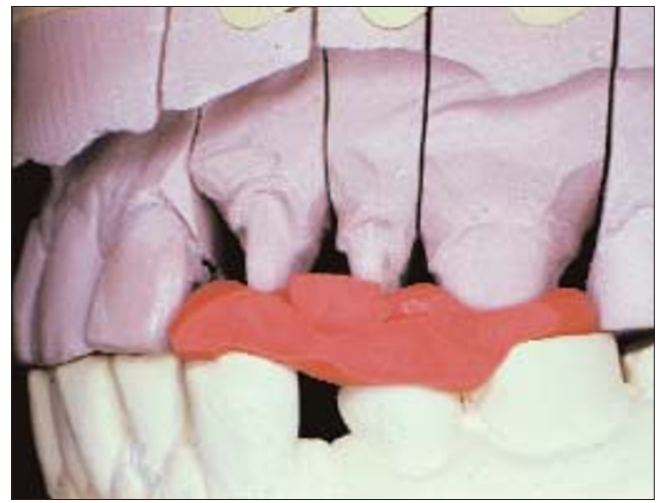

Fig. 13 A carefully trimmed interocclusal record restricted to the area of tooth preparation. The resulting crowns needed no adjustment

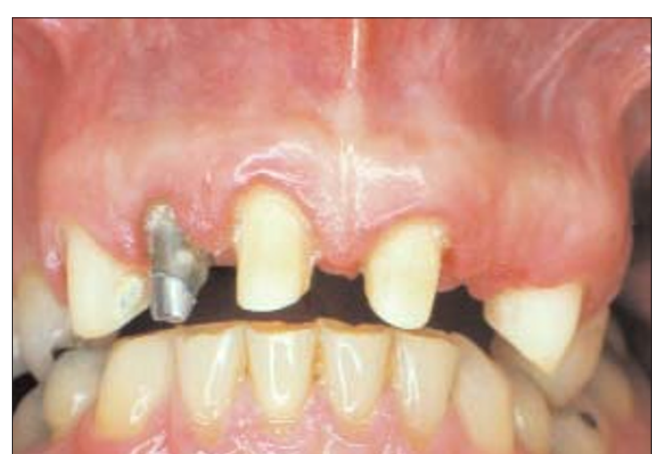

Fig. 14 Preparation of the teeth has resulted in loss of all guiding surfaces on the anterior teeth 
into (once set) a permanent record of the movements of the mandible (Fig. 15). When the working casts are articulated the acrylic guide table guides the articulator through the same movements that were present in the study casts, and the palatal surfaces of the upper teeth can be shaped to conform precisely to this. This technique is described in detail, with illustrations, in Reference 4.

The extra effort involved in using these techniques is not enormous, and where several anterior teeth are to be crowned we would strongly recommend using one or other of them.

1. Okeson JP. Management of temporomandibular disorders and occlusion. 1998. 4th Edition. St Louis: Mosby, pp47-51.

2. Posselt U. Studies in the mobility of the human mandible. Acta Odontol Scand 1952; $10: 109$.

3. Wise D. Occlusion and restorative dentistry for the general practitioner. Part 2- Examination of the occlusion and fabrication of study casts. Br Dent J 1982; 152: 160-165.

4. Howat A P, Capp N J, Barrett N V J. A colour atlas of occlusion and malocclusion. 1991. London: Wolfe Publishing, pp137139

5. Loos L G. Clinical criteria used to select an articulator. Compend Contin Educ Dent 1993; 14: 80-88.

6. Walls A W G, Wassell R W, Steele J G. A comparison of two methods for locating the intercuspal position (ICP) whilst mounting casts on an articulator. J Oral Rehabil 1991; 18: 4348.

7. Wise D. Occlusion and restorative dentistry for the general practitioner. Part 9 - Restoration of anterior teeth. Br Dent J 1982; 152: 407-413.

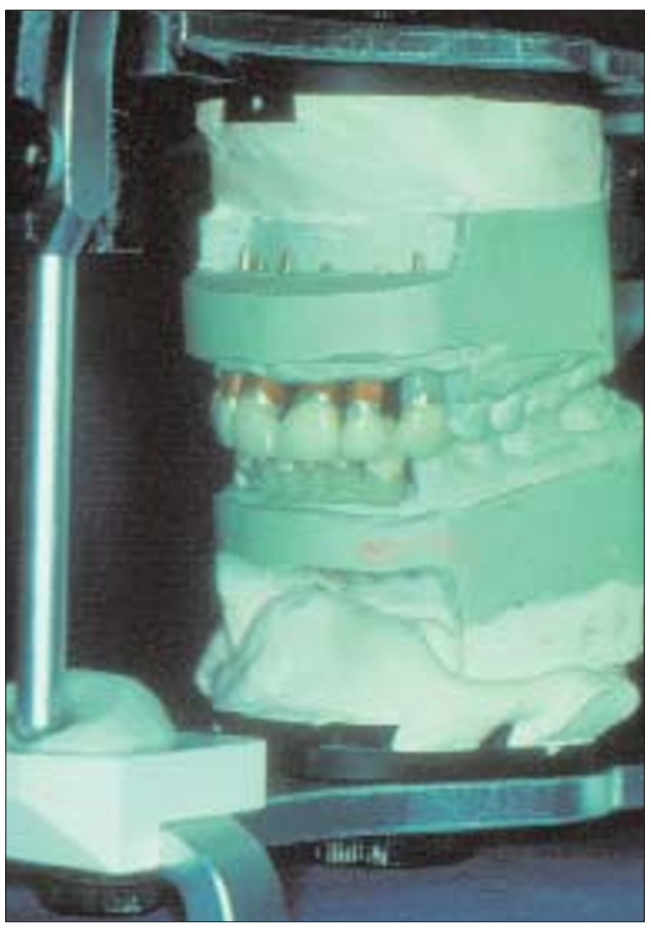

\section{Further reading}

1. Wise M. Occlusion and restorative dentistry for the general practitioner. Series of ten articles published in the Br Dent J 1982; 152: 117; 152: 160; 152: 197; 152: $235 ; 152: 277 ; 152$ $316 ; 152: 347 ; 152: 381 ; 152: 407$; 153: 13.

2 Howat A P, Capp N J, Barrett N V J. A colour atlas of occlusion and malocclusion. 1991. London: Wolfe Publishing.

3 Shillingburg H T Jr, Hobo S, Whitset L $D$, Jacobi R, Brackett SE. Fundamentals of fixed prosthodontics. 3rd ed. 1997. London: Quintessence Publishing Co.
Fig. 15 A custom acrylic guide table for use with a semi-adjustable articulator. This is an excellent method of copying tooth guidance into definitive restorations 